\title{
An Interesting Rendezvous with an Unexpected Complication in a Liver Transplantation Patient with Very Tight Anastomotic Stricture
}

\author{
Matar Khaled*1, Matar Mohammad*2, Khamaysi Iyad*3, Helal Ayman', Al Shami Salah*1, Abo JazrIyad*1, \\ Rosros Hassan*1, Al nahhal Mousa*1 and Abo Amouna Ashraf *1 \\ ${ }^{1}$ European Gaza hospital-Gastroenterology and hepatology department, Gaza Strip-Palestine.
}

${ }^{2}$ Al Shifa Hospital-Interventional Radiology department, Department Gaza Strip-Palestine.

${ }^{3}$ Rappaport Faculty of Medicine, Technion-Israel Institute of Technology, Haifa ,Israel.

Received: March 19, 2018; Published: April 06, 2018

*Corresponding author: Khaled Matar MD, Director, Invasive Endoscopy unit, Gastroenterology and hepatology department, European Gaza hospital, Gaza Strip-Palestine. Tel: 972592155123 ; Email: aboayman2001@hotmail.com

\section{Abstract}

An endoscopic or radiologic percutaneous approach may be an initial minimally invasive method for treating biliary strictures after living donor liver transplantation. However, cannulation of biliary strictures is sometimes difficult due to the presence of a sharp or twisted angle within the stricture or a complete stricture. The rendezvous technique can be used to overcome this difficulty. Herein, we report a modified rendezvous technique in for a patient with duct-to-duct anastomotic complete stricture after liver transplantation.

Keywords: Liver Transplantion; Bilo Pleural Fistula; ERCP; PTBD

Abbreviations: LDLT: Living Donor Liver Transplantation; ERCP: Endoscopic Retrograde Cholangio Pancreatography; PTBD: Percutaneous Transhepatic Biliary Drainage

\section{Case Summary}

\section{Clinical Scenario}

A 55-year-old male was referred to our unit with abdominal distention, jaundice, pancytopenia and fatigue for 2 months. He had been followed at the Gastroenterology clinic and had been worked thoroughly for pancytopenia, jaundice and splenomegaly [1]. Gastroscopy was carried out which showed grade 2 esophageal varices. Meanwhile he was diagnosed as having Hepatitis B related cirrhosis based on laboratory tests showing impaired hepatic synthetic and excretory functions along with positivity for hepatitis B surface antigen and detectable hepatitis DNA by quantitative PCR [2]. Then, upon further evaluation at the gastroenterology clinic he was found to have MELD score of more than 15 which qualifies him for listing for orthotopic liver transplantation. Meantime, he started having more abdominal discomfort mainly post parandial, early satiety, progressive abdominal distension and pedal edema for which he was put on diuretics [3]. He did not complain of neuropsychiatric symptoms suggesting hepatic encephalopathy. On examination, mild citrus and mild pedal edema; his blood pressure was 124/ 80 mm Hg PR: 90 bpm. Abdominal examination showed shrunken small liver, splenomegaly $(>4 \mathrm{~cm})$, and shifting dullness suggestive of Ascites. Investigations revealed haemoglobin of $9 \mathrm{gm} / \mathrm{dl}$, total leukocyte count of 3200 , platelet count of $65-105 /$,
INR: 2.1 Liver function tests revealed serum bilirubin of $5.4 \mathrm{mg} / \mathrm{dl}$, aspartame aminotransferase of $81 \mathrm{IU} / \mathrm{ml}$, alanine aminotransferase of $54 \mathrm{IU} / \mathrm{ml}$, alkaline phosphatise of 164 , and serum albumin levels of $2.5 \mathrm{~g} / \mathrm{dl}$ with globulins of $3.5 \mathrm{~g} / \mathrm{dl}$. Ascitic fluid analysis was wide gradient and there was no SBP. Her hepatitis B surface antigen was positive while ant hepatitis $C$ virus antibodies were negative [4].

An ultrasound Doppler revealed shrunken liver with coarsened echo texture $(13.6 \mathrm{~cm})$ and all three hepatic veins were patent. Also, there was no CBD dilatation and no intrahepatic biliary radicals dilatation. He had splenomegaly and splenic vein was $14 \mathrm{~mm}$ in diameter. There was moderate ascites and multiple spleno-renal collaterals were present. The final diagnosis was Hepatitis B related advanced liver cirrhosis Gastroscopy was carried out and showed evidence of Grade II Esopahgeal varices, mild portal hypertensive Gastropathy but no Fundal varices. After full evaluation he was referred for orthotopic liver transplantation. He was listed for OLT in Egypt, where he underwent the OLT successfully in March 2017 [5].

\section{Post Transplantation Course}

He came back to Gaza Strip in august 2017, where he was evaluated upon arrival in the gastroenterology clinic. His lab studies 
showed a dramatic improvement in hepatic synthetic and excretory functions with the following parameters detected; WBC's:4.5, Hgb: 11, Platelets: 220, AST:45, ALT: 40,ALP:157, Albumin:3.9, T.Bilirubin: 1.2, Tacrolimus Blood level: 9.3. He was maintained on the following postransplant medications: Mycophenolate Mofetil, Tacrolimus, Deptrin DS tab and valgancyclovir. Upon re-evaluation at follow up visit one month later, he was found to be jaundiced with disturbed liver function tests and T. Bilirubin of 7 [6]. MRI with MRCP was carried out which showed anastomotic tight stricture, dilated CBD proximal to the stricture, while distal to it close to ampulla the CBD is significantly attenuated, mild dilated intra hepatic biliary tracts, no stones or filling defect seen in itraoe extra hepatic biliary tracts.. He was admitted and kept on broad spectrum antibiotics, yet, he started having progressively deepening jaundice , intractable itching, fever and raising kidney function tests. Hence, the decision was to proceed to ERCP in order to avoid the fatal outcome of ascending cholangitis.
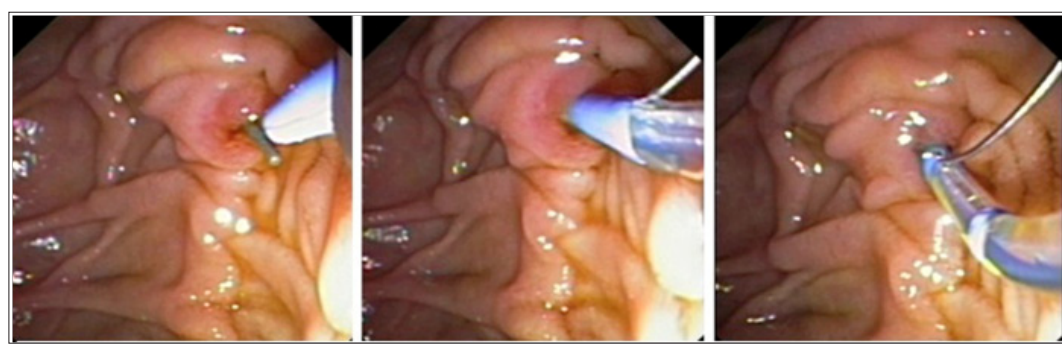

Figure 1: A Guidewire passing through the ampulla was captured by a snare and then withdrawn through the endoscopic working channel.

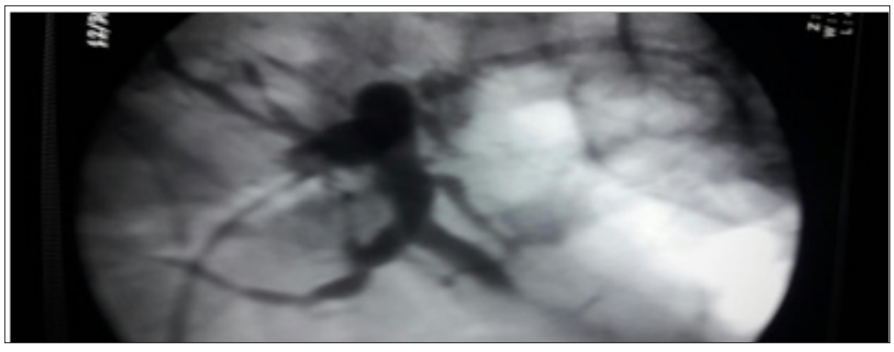

Figure 2: A tight Anastomotic stricture by percutaneous injection.

During ERCP cannulation was performed using standard method and accessories. The distal CBD was of a diameter of $0.8 \mathrm{~cm}$ and a tight stricture almost at the anastomosis where the guidewire could not be passed more proximally through the very tight stricture by any means [7]. So, the decision was to proceed with Rendezvous procedure with the aid of interventional radiologist. The PTC puncture was challenging because of the mild form of intra hepatic biliary tract, and high location of the transplanted liver in subcostal area, with the plan to avoid access through the costophernic recess. The percutaneous puncture was performed aided by ultrasound and access was gained into the mildly dilated right hepatic bile duct. The guidewire $(0.035)$ was introduced through the right intrahepatic duct and navigated through the anastomosis stricture, through the anastomosis, where after few trials the wire had been passed through the stricture into CBD and out of the ampulla of vater. Then, using the duodenoscope the wire coming out the ampulla of vater was pulled into the scope channel and a sphincterotome was passed over the guidewire into CBD then across the stricture successfully. Finally a plastic stent of $7 \mathrm{~F}^{*} 7 \mathrm{~cm}$ was inserted successfully through the stricture with good bile drain. The percutaneous rendezvous procedure enabled placement of an internal-external drain [8]. One week after the procedure the patient reported having right sided chest pain along with progressive shortness of breath. The chest Xray showed a massive right sided pleural effusion. CT of the abdomen showed the external catheter passing through the lower RT costophernic angle but with no lung injury or effect, it was believed that the high puncture through this the costophernic recess, in addition to possible inadequate internal drainage of the biliary system cause bile leak to pleural cavity and secondary large reactional pleural fluid accumulation, so the management was decided through drainage of the pleural fluid by using external drainage pleural catheter which revealed an exudative bilious fluid. then removal of the external drain followed by replacement of a longer new internal plastic stent placement. The right pleural fluid reacumulation was diminished significantly and the patients shortness of breath improved dramatically (Figures 1-4).

Figure 3: External Drain and biliary plastic Stent in
place.3/3




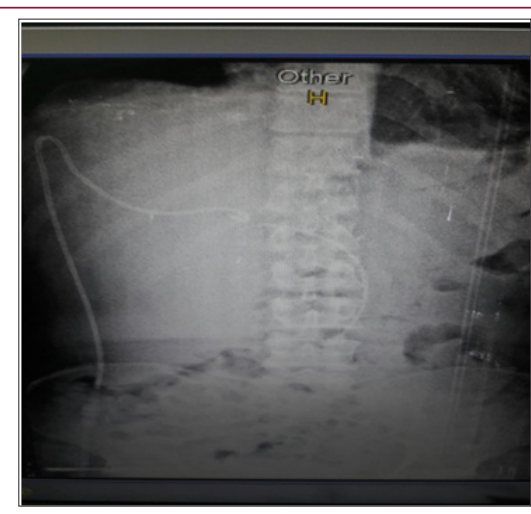

Figure 4: Post External and internal stent drain placement.

\section{Discussion}

Biliary strictures are not uncommon after living donor liver transplantation (LDLT), and two-thirds of biliary strictures can be treated by endoscopic retrograde cholangiopancreatography (ERCP). If ERCP fails, percutaneous transhepatic biliary drainage (PTBD) is recommended. Although maintaining a PTBD catheter for a long period is beneficial for treating biliary strictures, it has some disadvantages, as it cause decrease in quality of life of the patient as well as procedure related complications, such as leakage, pain, infection, and accidental removal of the PTBD catheter [9]. Therefore, permanent internal biliary drainage stent is preferred using percutaneous inserted stent or replacing PTBD catheters with endoscopically inserted stents. in our case, the external inserted plastic or metallic stents are not available in our area due to lack of supply, so decision to proceed Rendezvous technique through combined percutaneous puncture followed by passing the guide wire through the stricture to duodenum, then by endoscopic approach to pull the wire and use it to insert plastic stent endoscopically, this approach was chosen after the ERCP alone failed to bypass the wire through the CBD stricture. From our available data in our center, passing the guide wire through CBD stricture or narrowing is more applicable and carry more success rate that ERCP approach, this is due to easier torque and control of the guide wire, shorted distant to stricture, and variety of wires and catheters that can be used to asses passage through the stricture.

Though all precaution were taken by using combined US and fluoroscopy guidance to avoid unexpected complications such as injury to the right pleural costophrenic reflection, such complication still happening and un avoidable especially in liver transplant patient were the location of the liver is high in subcostal area , in addition due to deep inferior location of the costo-phernic recess with no lung tissue or aeration inside it in these patients.

Injury during PTBD placement resulting in a bilo-pleural fistula is a rare complication. It can be managed by draining the pleural fluid by means of paracenthesis using chest tube or catheter drainage, in addition to insuring appropriate bile drainage by repeating PTBD drainage after removing the initial catheter. In this context, the decision was to deal with the bilo-peural fistula in the same manner as the postcholecystectomy bile leaks by insuring good bile drainage through the endoscopically inserted plastic stent and good drainage of the pleural fluid, in a manner similar to management of a bile leak, a leak to a cavity other than the abdominal cavity can be managed by facilitating spontaneous bile flow through the natural ways while allowing time for spontaneous healing of the iatrogenic fistulous tract.

\section{Conclusion}

Bile drainage can be achieved by means of a combined percutaneous transhepatic and endoscopic transpapillary approach (the rendezvous technique). Rendezvous procedure is when PTC is used to access the biliary tree in an anterograde fashion facilitating successful completion of a challenging ERCP. Randevue procedure is based upon liaison between the endoscopist and the interventional radiologist and it is a cornerstone step in achieving access into the biliary system and certain kinds of stricture when the transpapillary access or access through a tight stricture could not be achieved. Ile leak or fistula as a procedure complication can be managed in a timely manner endoscopically in a fashion similar to post cholecystectomy ile leak with sphincterotomy and stenting to facilitate bile drainage through natural route permitting healing of the leak site or the bilopleural fistula.

\section{Acknowledgment}

We would like to extend our sincere appreciation to Dr. Mohammed Matar the interventional radiologist for their assistance in the success of the randevues procedure and also creation of this manuscript.

This research did not receive any specific grant from funding agencies in the public, commercial, or non-for-profit sectors.

\section{References}

1. Yazumi S, Yoshimoto T, Hisatsune H, Hasegawa K, Kida M, et al. (2006) Endoscopic treatment of biliary complications after right-lobe livingdonor liver transplantation with duct-to-duct biliary anastomosis. J Hepatobiliary Pancreat Surg 13(6): 502-510.

2. Kim ES, Lee BJ, Won JY, Choi JY, Lee DK (2009) Percutaneous transhepatic biliary drainage may serve as a successful rescue procedure in failed cases of endoscopic therapy for a post-living donor liver transplantation biliary stricture. Gastrointest Endosc 69(1): 38-46.

3. Sharma S, Gurakar A, Jabbour N (2008) Biliary strictures following liver transplantation: past, present and preventive strategies. Liver Transpl 14(6): 759-769.

4. Aytekin C, Boyvat F, Yimaz U, Harman A, Haberal M (2006) Use of the rendezvous technique in the treatment of biliary anastomotic disruption in a liver transplant recipient. Liver Transpl 12(9): 1423-1426.

5. Chang JH, Lee IS, Chun HJ, Choi JY, Yoon SK, et al. (2010) Usefulness of the rendezvous technique for biliary stricture after adult right-lobe livingdonor liver transplantation with duct-to-duct anastomosis. Gut Liver 4(1): 68-75.

6. Fiocca F, Salvatori FM, Fanelli F, Bruni A, Ceci V, et al. (2011) Complete transection of the main bile duct: minimally invasive treatment with an endoscopic-radiologic rendezvous. Gastrointest Endosc 74(6): 13931398.

7. Ödemiş B, Shorbagi A, Köksal AȘ, Özdemir E, Torun S, et al. (2013) The "Lasso" technique: snare-assisted endoscopic-radiological rendezvous technique for the management of complete transection of the main bile duct. Gastrointest Endosc 78(3): 554-556.

8. Dowsett JF, Vaira D, Hatfield AR, Cairns SR, Polydorou A, et al. (1989) Endoscopic biliary therapy using the combined percutaneous and endoscopic technique. Gastroenterology 96(4): 1180-1186. 
9. Chang JH, Lee IS, Chun HJ, Choi JY, Yoon SK, et al. (2010) Usefulness of the rendezvous technique for biliary stricture after adult right-lobe living- donor liver transplantation with duct-to-duct anastomosis. Gut Liver $4(1)$ : 68-75. (c) (i) This work is licensed under Creative

Submission Link: https://biomedres.us/submit-manuscript.php

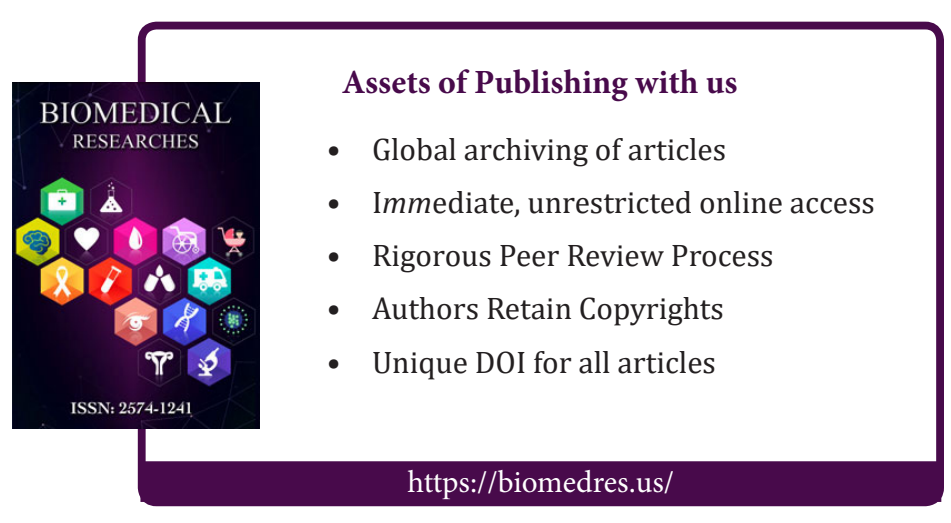

